\title{
ENSINO CLÍNICO NA COMUNIDADE (*)
}

\author{
CLINICAL TEACHING IN COMMUNITY HEALTH SERVICES
}

\author{
Luiz Ernesto de Almeida Troncon
}

\begin{abstract}
Docente do Departamento de Clínica Médica; Ex-Presidente da Comissão de Graduação, Faculdade de Medicina de Ribeirão Preto da Universidade de São Paulo

Correspondência: Dr. Luiz E. A. Troncon - Departamento de Clínica Médica - Hospital das Clínicas, Campus da U.S.P. - 14048-900 Ribeirão Preto, SP - Telefone: (16) 633-0436 - Fax: (16) 633-6695 - e-mail: ledatron@fmrp.usp.br
\end{abstract}

TRONCON LEA. Ensino clínico na comunidade. Medicina, Ribeirão Preto, 32: 335-344, jul./set. 1999.

RESUMO: No curso de graduação em Medicina a mudança do eixo do ensino clínico do hospital universitário para a rede de serviços de saúde na comunidade, constitui, atualmente, uma forte tendência no campo da Educação Médica. Busca-se, com isto, a construção de um novo paradigma de atuação médica, mais adequado às necessidades do público. Esta proposição apresenta justificativas conceituais válidas, tanto do ponto de vista médico como do educacional, e oferece vantagens potenciais para o aprendizado dos estudantes. A proposta de mudança sofre, porém, no interior das escolas médicas, resistências de ordem variada, sobretudo de ordem cultural; a sua implementação encontra, ainda, inúmeras dificuldades práticas. A superação destes obstáculos constitui uma difícil tarefa, que deve envolver não só a escola médica, mas, também, o governo e as entidades médicas. Medidas efetivas de apoio ao ensino clínico na comunidade devem contemplar o fortalecimento do sistema nacional de saúde e a valorização do trabalho dos médicos junto à comunidade.

UNITERMOS: Estudantes de Medicina. Escolas Médicas. Redes Comunitárias. Serviços de Saúde Comunitária. Educação Médica.

\section{INTRODUÇÃO}

Nos últimos anos, a importância do desenvolvimento de parte substancial do ensino de graduação em Medicina, na área clínica, na rede de serviços de saúde da comunidade tem sido crescentemente salientada. Esta tendência, cuja origem remonta já às primeiras décadas deste século, durante longo tempo, constituiu iniciativa isolada de alguns departamentos que, dentro das escolas médicas, tinham atuação mais voltada aos aspectos sociais da Medicina. No entanto, mais recentemente, a proposta de retirar do hospital universitário uma parte considerável do ensino clínico passou a integrar propostas mais abrangentes de reformas na Educação Médica ${ }^{(1,2)}$.
Neste artigo, pretende-se apresentar, de forma breve, algumas das justificativas para a proposição de deslocar o eixo do ensino clínico de graduação em Medicina, do hospital para os serviços de saúde disponíveis na comunidade, bem como discutir, sucintamente, algumas das vantagens potenciais da mudança. Pretende-se, também, tecer comentários sobre as principais fontes de resistência à aceitação deste conceito e sobre as dificuldades práticas que limitam sua implementação no atual contexto brasileiro.

\section{JUSTIFICATIVAS}

A principal justificativa da proposta de desenvolver parte considerável do ensino clínico junto à 
comunidade consiste na busca da formação de médicos com um perfil de competências profissionais mais adequado às necessidades da sociedade como um todo. Fica implícito que o paradigma de atuação médica vigente, na maior parte das sociedades contemporâneas, não tem maior compromisso com esta adequação. De fato, já no início dos anos setenta, o saudoso Professor Hélio Lourenço de Oliveira observava:

..."A profissão médica, sob o impacto dos avanços contemporâneos dos meios de diagnóstico, de tratamento e da cirurgia, concentra-se mais e mais em hospitais e clínicas, no exercício de uma medicina curativa de eficiência crescente, mas às custas de perder progressivamente o contacto com a comunidade" (3).

No conceito citado, está implícito que o sistema vigente de formação dos médicos é, ao menos em parte, responsável por este distanciamento. Enquanto que, em muitos países, procurou-se, a partir da segunda metade deste século, adequar o sistema de serviços de saúde aos interesses públicos, não houve preocupação semelhante com relação à atuação das escolas médicas. Isto é bem ilustrado pela impressão de outra autoridade médica de expressão internacional, o Dr. Hafdan Mahler, Diretor Geral da Organização Mundial de Saúde, que comentava em 1977.

"Ao observador atento das escolas de Medicina, parecerá inquietante perceber a regularidade com que todo o sistema educacional destas escolas está isolado em relação aos sistemas de serviço de saúde de seus países..." (4).

Justifica-se, por conseguinte, a construção de um novo modelo de exercício da Medicina que, além de melhor atender às necessidades da comunidade, coadune-se com os ideais humanitários da profissão médica. No que se refere ao engajamento da escola médica na consecução deste objetivo, a análise racional do problema e das alternativas possíveis recomenda que a melhor estratégia é o desenvolvimento em maior escala de atividades externas. Neste sentido, na mesma época, assim se expressava o Professor Hélio Lourenço:

"O trabalho médico no hospital dificilmente pode ser considerado, do ponto de vista educacional, como uma amostragem adequada dos problemas médicos existentes. Os estudantes devem, nas fases precoces do seu treinamento, serem ex- postos à experiência extramural, para se familiarizarem com as manifestações iniciais da doença, bem como com toda a evolução das doenças mais comuns.... Ademais, o estudante deveria não só aprender a reconhecer as doenças e tratar os pacientes.Todas as condições ambientais conducentes à doença ou facilitadoras do seu desenvolvimento deveriam se vividamente trazidas à compreensão do estudante de medicina. A prevenção das doenças e a promoção de uma saúde melhor deveriam também ser sua preocupação"(3).

A inadequação do paradigma tradicional de atuação médica, desenvolvido ao longo deste século, motivou a proposição de um modelo alternativo, voltado mais à promoção da saúde do que à cura das doenças (Tabela I). Neste modelo inovador, a maior parte das ações são desenvolvidas junto à própria comunidade e não no hospital, de modo a contemplar prática médica mais abrangente e menos especializada. Assim, o exercício da profissão deve depender menos de recursos tecnológicos e mais das habilidades clínicas fundamentais, que demandam maior interação do médico com os pacientes. Desta maneira, criam-se condições para uma prática mais humanizada e mais orientada para a pessoa e o seu meio de inserção familiar e social ${ }^{(5)}$. Seu caráter é, fortemente, de prevenção das doenças e de promoção da saúde, mais do que da busca da cura das moléstias, o que constitui o principal determinante da atuação profissional dentro do paradigma tradicionalmente vigente.

A proposta de desenvolver parte considerável do ensino clínico, no curso de graduação em Medicina, nos serviços de saúde da comunidade, significando, portanto, um maior engajamento da escola médica em atividades extramurais, integra, também, um novo

\begin{tabular}{|ll|}
\hline $\begin{array}{l}\text { Tabela I - Enfoques de diferentes } \\
\text { prática médica }\end{array}$ & Inovadigmas da \\
\hline Tradicional & Saúde \\
\hline Doença & Comunitário \\
Hospitalar & Abrangência \\
Especialização & Humanização \\
Tecnologia & Prevenção \\
Cura & Comunidade \\
Indivíduo & \\
\hline
\end{tabular}


paradigma educacional ${ }^{(1)}$. No modelo tradicional de Educação Médica (Tabela II), o foco do desenvolvimento das atividades de ensino e aprendizagem é o professor. É ele quem determina, com exclusividade quase absoluta, o que vai ser ensinado e como deve o aluno aprender. Ao contrário, o novo paradigma é fortemente centrado no estudante, que tem maior liberdade para definir os seus objetivos instrucionais e, principalmente, para selecionar os recursos que deve utilizar para compor o seu aprendizado. A isto se refere a afirmação, que vem se tornando um lugar comum, de que o estudante deve, no curso de graduação, "aprender a aprender" Medicina ${ }^{(1,2)}$. Isto implica em uma mudança radical no papel do professor, que passa a atuar mais como um "facilitador" do aprendizado do estudante, do que exercer o papel tradicional de transmissor do conhecimento.

\begin{tabular}{|ll|}
\hline \multicolumn{2}{|l|}{$\begin{array}{l}\text { Tabela II - Enfoques de diferentes paradigmas da } \\
\text { educação médica }\end{array}$} \\
\hline Tradicional & Inovador \\
\hline Ensino (Professor) & Aprendizado (Aluno) \\
$\begin{array}{l}\text { Conhecimento } \\
\text { (Habilidades) }\end{array}$ & $\begin{array}{c}\text { Habilidades e Atitudes } \\
\text { (Conhecimentos) }\end{array}$ \\
$\begin{array}{l}\text { Reunião de Fatos } \\
\text { Disciplinas/Cursos }\end{array}$ & $\begin{array}{c}\text { Integração de Informações } \\
\text { Programa Padrão }\end{array}$ \\
Elesproblemas \\
\hline
\end{tabular}

O desenvolvimento do processo de aprendizado, no novo modelo, baseia-se, predominantemente, no estudo de tópicos gerados na tentativa de busca de soluções para os problemas concretos que são apresentados aos estudantes, mais do que no desenvolvimento de cursos ou disciplinas individualizados. Desta maneira, o conhecimento adquirido pelo estudante constrói-se pela integração de dados relevantes, obtidos dentro de um contexto apropriado, e não pelo simples acúmulo de informações de importância discutível, não necessariamente pertinentes a um arcabouço lógico. Um método fundamental para a operacionalização deste novo conceito educacional é o que se denomina de "Problem-based Learning"(6), que vem se constituindo no esteio principal das reformas curriculares, levadas a cabo com resultados altamente satisfatórios, em escolas médicas do maior prestígio no Hemisfério Norte ${ }^{(7,8)}$.
O modelo tradicional de ensino médico tem, como uma de suas principais características, o compromisso formal com a transmissão do conhecimento nas áreas tradicionais das Ciências Básicas e Clínicas. Isto vem sendo progressivamente dificultado pelo crescimento, em ritmo avassalador, da massa de conhecimento disponível ${ }^{(9)}$. Na tentativa de acompanhar a expansão das fronteiras do conhecimento em cada área, as escolas médicas acabam por sobrecarregar suas estruturas curriculares com atividades compulsórias, voltadas à transmissão de informações factuais. Além disso, o conhecimento novo, muito freqüentemente, apresenta-se na forma de inovações tecnológicas, voltadas ao diagnóstico ou ao tratamento de doenças específicas; disto resulta a tendência à maior exposição dos estudantes a procedimentos baseados em recursos tecnicamente mais sofisticados ${ }^{(10)}$. Este impulsionamento dado ao ensino médico frequientemente resulta em menor destaque ao aprendizado de habilidades clínicas básicas. Esta distorção foi muito bem apreendida pelo Dr. Stephen Muller, da Johns Hopkins Medical School, que, em 1983, observou:

"As escolas médicas vêm submergindo os estudantes em pormenores opressores sobre conhecimentos especializados e aplicação de tecnologia sofisticada, restringindo a aprendizagem de habilidades médicas fundamentais; isto pode levar a uma fascinação pela tecnologia, tornando o artefato mais importante que o paciente..." (10).

O novo modelo educacional diferencia-se, ainda, do tradicional na medida em que reconhece diferenças individuais importantes no interesse dos estudantes e, principalmente, nos estilos de aprendizagem $^{(11)}$. Reconhecendo, também, a impossibilidade de responder pela cobertura de todo o campo de atuação médica, oferece a possibilidade do desenvolvimento de maior número de atividades opcionais, em contraposição a maior carga de atividades compulsórias, constantes do modelo tradicional de ensino médico.

Uma característica marcante do ensino tradicional é a valorização expressiva dos aspectos cognitivos do desempenho do estudante, em detrimento do reconhecimento do domínio das habilidades clínicas fundamentais ou do desenvolvimento de atitudes positivas frente a diferentes aspectos da prática médica.

A busca de correções para estas distorções tem levado a propostas de reformulação do ensino médico, que se caracterizam pela valorização da aquisição das habilidades médicas mais gerais, e ao desenvolvi- 
mento de padrões de atitudes mais adequados. Coloca-se, assim, maior ênfase no domínio do método clínico, na capacidade de comunicação com as pessoas e na atitude de compreensão e aceitação dos aspectos sociais, da importância da promoção da saúde, da reabilitação e da investigação científica em Medicina ${ }^{(1,2)}$.

Neste contexto de mudanças, o desenvolvimento de atividades clínicas na comunidade pode ser visto como altamente favorável à aquisição de conhecimento vinculado a problemas reais de saúde, inseridos em situações pessoais, familiares e sociais realistas. Pode, também, ser apropriado à aquisição de habilidades clínicas básicas e ao desenvolvimento dos padrões de atitudes necessários à atuação em um novo modelo de exercício da Medicina ${ }^{(1,2,12,13)}$.

Um outro conjunto poderoso de forças favoráveis ao desenvolvimento de atividades de ensino clínico fora dos muros do hospital universitário é de natureza mais prática do que conceitual. Estas forças são geradas pelo reconhecimento crescente de que o hospital de ensino tradicional vem se tornando inadequado ao ensino médico de graduação e ao exercício de habilidades clínicas fundamentais. A hierarquização dos diferentes níveis de assistência médica, necessária à organização eficiente dos sistema de saúde, bem como a inevitável inserção da quase totalidade dos hospitais de ensino neste sistema, resulta em que estes hospitais passem a congregar casos mais complexos ou de maior gravidade. Por outro lado, o alto custo de manutenção obriga a que a permanência hospitalar seja restrita ao mínimo necessário; isto, freqüentemente, dificulta, ou mesmo, inviabiliza a inclusão dos pacientes internados em atividades de ensino. Além disso, o desenvolvimento do ensino clínico e a supervisão das atividades dos estudantes no ambiente hospitalar fica, naturalmente, a cargo de pessoal docente, fortemente requisitado nas tarefas de assistência médica especializada, na orientação dos programas de Residência Médica ou de Pós-Graduação "sensu strictu", ou de investigação científica. Com isto, estabelece-se inexorável competição na utilização do tempo e da energia do professor que, invariavelmente, resulta em detrimento do ensino em nível de graduação.

\section{VANTAGENS POTENCIAIS}

A alocação de parte considerável do tempo do estudante a atividades educacionais a serem desenvolvidas nos serviços de saúde voltados à comunidade apresenta um número de vantagens potenciais de natureza diversa. Diferentemente do ambiente hospi- talar, os casos clínicos, têm maior probabilidade de serem mais adequados às finalidades de ensino, no que se refere à sua natureza, grau de complexidade e representatividade do que ocorre na população em geral. Isto é particularmente importante em um modelo educacional inovador, onde a fonte principal da geração de objetivos instrucionais é a análise de problemas reais e concretos. Conforme ilustra a Figura 1, o nível correspondente ao ensino hospitalar e, em menor extensão, ao ambulatorial, permitem a exposição do estudante a um contingente quantitativamente menor e mais particularizado de problemas de saúde; o doente internado no hospital oferecerá, obviamente, experiência educacional centrada predominantemente na doença instalada e em suas complicações, bem como em aspectos peculiares do manejo clínico. Ao contrário, a atuação do estudante, nos serviços de saúde da comunidade, pode propiciar o contato com um espectro mais amplo e mais numeroso de casos, e a oportunidade de identificação das manifestações iniciais das doenças. Além disso, a exposição do estudante de Medicina aos casos, problemas e situações de relevância médico-sanitária, vistos na comunidade, processa-se dentro do contexto em que eles efetivamente existem. Será possível, portanto, vivenciar as repercussões dos problemas de saúde na vida pessoal e profissional da pessoa acometida, bem como em sua família e no meio social em que se insere; isto permite, evidentemente, visão mais ampla e completa da realidade médica e social. Mais ainda, criam-se condições para o entendimento apropriado das condições ambientais e sociais, responsáveis pela produção das doenças ou favorecedoras do seu desenvolvimento, assunto do qual nos falava o Professor Hélio Lourenço de Oliveira ${ }^{(3)}$, e anteriormente mencionado.

É, também, plausível que o engajamento do estudante em atividades próprias de uma rede de serviços de saúde, suficientemente ampla e com adequada diversificação, ofereça maiores e melhores oportunidades de aprendizado e do exercício das habilidades básicas que compõem o método clínico, bem como das diferentes práticas úteis a todos os médicos. Pode, ainda, propiciar a aquisição de algumas habilidades muito específicas, como a de trabalhar harmoniosamente em equipes integradas por outros profissionais de áreas diversas, bem como o desenvolvimento de qualidades pessoais altamente vantajosas, como, por exemplo, a capacidade de liderança, a iniciativa e a eficiência na busca de informações necessárias, autoaprendizado constante. Igualmente, admite-se que o ensino clínico na comunidade possa propiciar condições 


\section{PROBLEMAS DE SAÚDE DOS PACIENTES: DIFERENTES PONTOS DE VISÃO}

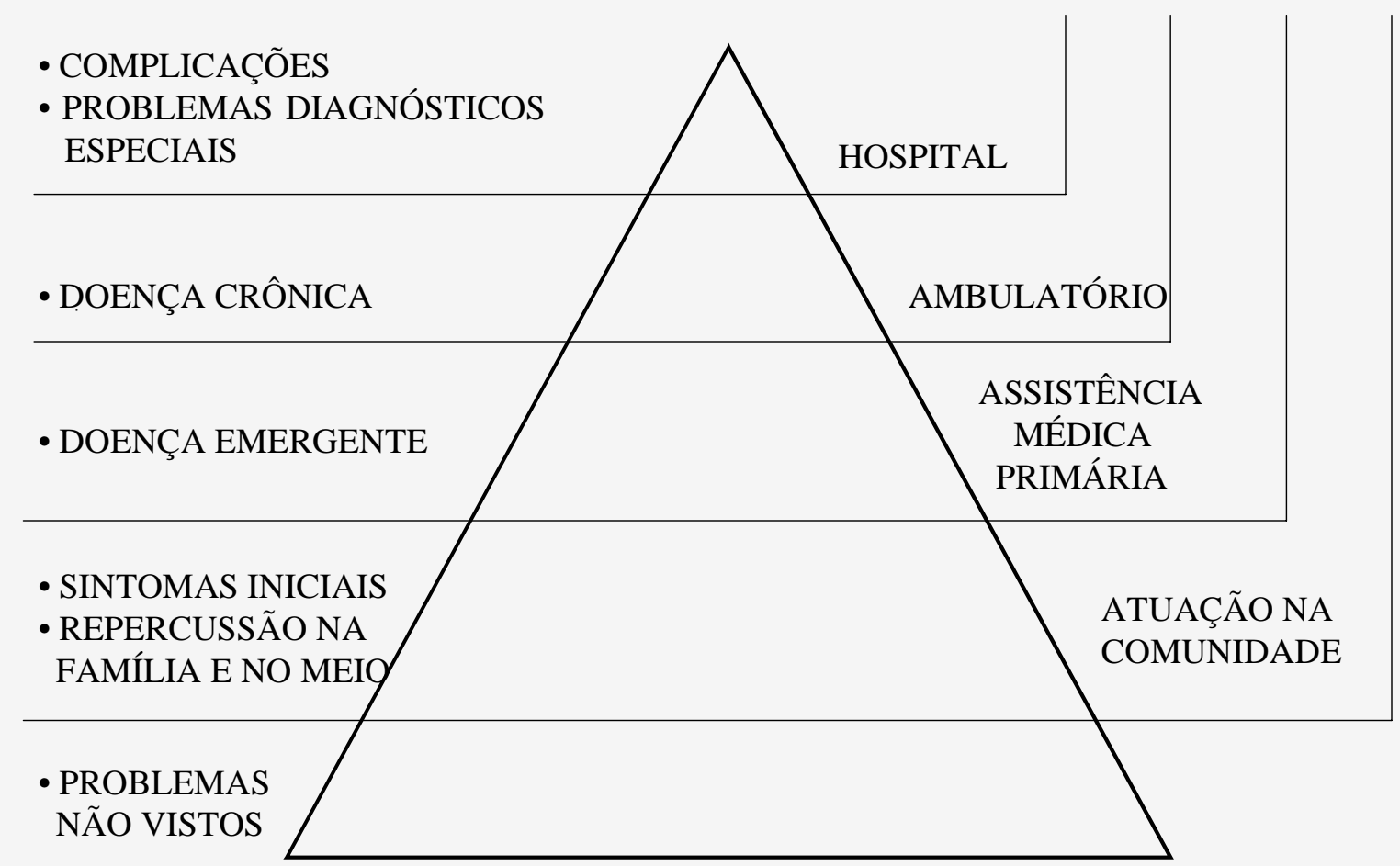

Figura 1 - Os problemas dos pacientes segundo diferentes pontos de visão da profissão médica (modificado de Harden, Snowden \& Dunn $\left.{ }^{(1)}\right)$.

mais favoráveis para o desenvolvimento de atitudes positivas frente a vários aspectos da atuação médica, o que é requisito indispensável à construção do novo paradigma de exercício da Medicina (Tabela I).

Por fim, a atuação do estudante junto à comunidade pode propiciar melhores condições para a aquisição de noções e conceitos sobre o sistema e os serviços de saúde oferecidos ao público; é provável que o estudante adquira experiência pessoais relevantes e conheça, de forma efetiva, as condições reais de funcionamento do sistema nacional de saúde.

Cumpre-se ressaltar, entretanto, que as vantagens potenciais do ensino clínico na comunidade, acima apresentadas, apesar de plausíveis, constituem, em grande parte, pressupostos teóricos. A comprovação de que um novo modelo de Educação Médica, com as características sumariadas anteriormente (Tabela II), e que, junto com outras mudanças, contemple o desvio do eixo do aprendizado clínico do hospital para a comunidade, trará, de fato, estas vantagens depende da implantação, em larga escala, deste novo paradigma. Além disso, a comprovação dependerá da adequada avaliação do perfil de conhecimentos, habilidades e atitudes dos graduandos formados no novo modelo. Isto constitui, obviamente, tarefa de enorme complexidade, mas os relatos dos centros pioneiros, que se engajaram neste novo paradigma ${ }^{(7,8)}$, bem como as experiências preliminares das instituições que mantiveram turmas cursando, paralela e simultaneamente, os modelos tradicional e inovador ${ }^{(8,14)}$, apontam para resultados animadores.

Deve-se considerar, entretanto, que as vantagens potenciais, acima apresentadas, dependem de alguns requisitos básicos. São eles relacionados à estruturação e ao funcionamento da rede de serviços, às características do pessoal disponível, notadamente o pessoal médico, bem como à articulação entre os serviços de saúde na comunidade e a escola médica (Tabela III). A capacidade física da rede de serviços deve ser suficientemente ampla para acomodar o número, em geral elevado, de estudantes de Medicina de cada região geográfica. É desejável que os estudantes se distribuam pelas unidades de modo que cada uma delas abrigue um número muito reduzido. 
Tabela III - Requisitos básicos para o desenvolvimento do ensino clínico na comunidade

- REDE DE SERVIÇOS:

- Ampla

- Organizada

- Eficiente

- Ações diversificadas

- PESSOAL

- Número adequado

- Capacitação Técnica

- Capacitação Educacional

- Modelos de atitude adequados

- Motivação

- VÍNCULO ESTÁVEL COM A UNIVERSIDADE

- Orçamentário

- Administrativo

- Educacional

Além de organizadas e eficientes, as unidades devem oferecer um espectro amplo e diversificado de ações abrangentes de saúde, que transcendam com clareza, a prestação de serviços de diagnóstico e tratamento de doenças de menor complexidade e que incidam sobre o ambiente físico e os fatores sociais relacionados à saúde.

Em especial, a rede de serviços de saúde na comunidade deve dispor de pessoal numeroso, que detenha capacitação e motivação para a adequada supervisão dos estudantes. O número de pessoas disponíveis, bem como o tamanho da rede de serviços, em cada região geográfica, podem ser considerados determinantes maiores da qualidade das atividades educacionais a serem desenvolvidas pelos estudantes. O pessoal médico deve, idealmente, dispor de capacitação técnica para o exercício de funções médicas gerais, na comunidade, bem como de treinamento adequado para a supervisão das atividades educacionais. Em especial, o contato com os estudantes deve ficar a cargo daqueles profissionais que sabidamente detenham um perfil de padrões de atitudes adequado, de modo a que possam funcionar como "role models" apropriados para os estudantes neles se espelharem ${ }^{(15)}$.
O bom desenvolvimento do ensino clínico na comunidade requer, por fim, a existência de vínculos estáveis entre a Universidade e a Comunidade, que inclua a manutenção de canais constantemente abertos entre as instâncias competentes da escola médica e dos serviços de saúde. Isto pode ser conseguido pela constituição de um foro único, com representação das duas partes e com a inclusão dos estudantes, que tenha a incumbência de abordar os diferentes aspectos orçamentários e administrativos, envolvidos nesta interação, e que cuide do provimento de capacitação educacional ao pessoal responsável pela supervisão dos estudantes.

\section{BARREIRAS À ACEITAÇÃO}

A mudança de um modelo tradicional de formação de médicos para outro, de características inovadoras (Tabelas I e II), enfrenta um número de barreiras à sua aceitação. Grande parte das resistências ao deslocamento do ensino clínico do hospital para a comunidade são de ordem cultural e, aparentemente, estão presentes, em maior ou menor grau, nas diferentes instituições envolvidas nas mudanças. Consideraremos, aqui, algumas das que julgamos reconhecer no seio da escola médica e da Universidade.

A maioria das pessoas reunidas nos órgãos que tem a responsabilidade por decisões que conduzem a transformações relevantes, tem, como posições inerciais naturais, a resistência à mudança e o temor ao novo. A superação do conservadorismo é dificultada pelo preconceito e desinformação, especialmente, nos meios acadêmicos e médicos, a de valores peculiares a certas "culturas profissionais" $(14,16 / 19)$. No meio acadêmico brasileiro, fatores de ordem cultural determinam que o desenvolvimento de atividades de investigação científica tenha maior valor intrínseco que o de atividades educacionais e o ensino de pós-graduação seja mais valorizado que o de graduação. Por outro lado, no meio médico, em particular, desenvolveu-se uma escala de valores na qual o especialista tem mais prestígio que o médico geral, o uso de equipamentos de alta tecnologia é visto como mais confiável que a aplicação de métodos clínicos tradicionais e o trabalho no hospital é melhor conceituado que o exercido nas unidades básicas de saúde ${ }^{(14)}$. A formação destes valores, evidentemente, não se fez ao acaso, mas obedeceu à lógica da inserção da Medicina em um paradigma positivista e mecanicista ${ }^{(16)}$, que associa a eficácia na área da saúde à aplicação de alta tecnolo- 
gia na busca da solução de problemas individuais de natureza orgânica. Vale frisar que, neste sentido, a cultura profissional médica apenas reproduz o que Habermas denomina de "ideologia do especialista profissional", que enfatiza a racionalidade tecnológica e instrumental, em consonância com interesses maiores, de natureza industrial, burocrática ou militar de uma dada sociedade ${ }^{(16)}$.

Assim sendo, propostas que, dentro da escola médica, impliquem em investimento de tempo, energia e recursos para mudanças no ensino de graduação, que conduzam a maior valorização de atividades extra-hospitalares menos especializadas e mais voltadas à comunidade, serão, possivelmente, de difícil aceitação.

Agrava este quadro o tipo de estrutura organizacional e de poder de decisão prevalente na Universidade, em geral, e na escola médica em particu$\operatorname{lar}^{(18,19)}$. Esta pode ser considerada como uma federação de departamentos autônomos muito fortes, cujos interesses podem sobrepor-se aos da escola médica como um todo. Cada departamento, por sua vez, constitui outra federação de grupos, disciplinas ou especialidades com metas próprias e que nem sempre coincidem com os objetivos mais gerais da escola ou da Universidade.Deste modo, o sucesso de propostas de mudança depende da sua capacidade de atender não só aos interesses maiores da escola médica como um todo, mas, também, os de indivíduos e os dos grupos ou setores que se congregam nos departamentos. Por outro lado, estes tendem a se orientar mais por desígnios subordinados às respectivas culturas profissionais do que pelas metas institucionais gerais.

Outra importante barreira à introdução de mudanças mais profundas na educação médica é a insegurança quanto à qualidade do médico que virá a se formar. Isto decorre, em grande parte, do conceito equivocado que o distanciamento do hospital, onde se sediam os departamentos, irá associar-se à perda da qualidade do saber adquirido. Provavelmente, um determinante deste sentimento de insegurança é a noção da perda de controle do processo educacional, que poderá passar a se concentrar mais nas mãos dos estudantes e dos preceptores de atividades clínicas extramurais do que na dos professores baseados nos departamentos acadêmicos.

Mesmo em ambientes, onde prevaleçam condições mais favoráveis à aceitação de um novo modelo educacional, pode existir um outro fator restritivo, próprio do momento atual da Universidade Brasileira, que é a falta de incentivo individual para o envolvimento em processos de transformação. Isto decorre da insatisfação geral quanto às condições de trabalho e de remuneração, bem como da percepção de que maior investimento em mudanças educacionais ou em atividades extramurais não será devidamente reconhecido em julgamentos relacionados à promoção na carreira.

No contexto brasileiro contemporâneo, emerge um outro fator de resistência que pode afetar particularmente o conjunto de estudantes, preocupados, é natural, com sua inserção futura em um mercado de trabalho médico fortemente determinado pelo paradigma tradicional de medicina especializada. De modo análogo ao que ocorre em outros ramos da atividade humana em países sul-americanos, o mercado brasileiro de trabalho médico sofreu influência marcante do que ocorreu em décadas passadas nos Estados Unidos da América. Naquele país, diferentemente do que ocorre na Inglaterra ou no Canadá, a proporção de especialistas na prática médica cresceu de forma a ser, atualmente, muito superior a de médicos genera$\operatorname{listas}^{(20,21)}$. Refletindo as condições dos Estados Unidos, o mercado brasileiro caracteriza-se, hoje, por oferecer melhores oportunidades de trabalho, de prestígio profissional e de remuneração aos especialistas e, em particular, àqueles que detêm o domínio de métodos diagnósticos ou terapêuticos dependentes de equipamento de alta tecnologia. Além disso, falta, no sistema de saúde vigente no país, e no mercado de trabalho médico, em geral, a posição profissional bem definida de um "general practioner", que receba não só remuneração adequada, como também reconhecimento profissional. $\mathrm{O}$ papel que caberia a este tipo de médico é desempenhado por mão-de-obra médica não completamente qualificada para atividades gerais na comunidade e que tem, freqüentemente, caráter temporário. Esta situação dificulta sobremaneira a implantação de programas de treinamento pós-graduado de não especialistas e a valorização, pelos próprios estudantes de graduação, de atividades clínicas extramurais. De fato, a maioria dos estudantes aspira a trabalhar, no futuro, em ramos especializados da Medicina; em levantamento recente, efetuado entre os graduandos da Faculdade de Medicina de Ribeirão Preto, verificou-se que menos de $10 \%$ dos mesmos tencionava trabalhar, no futuro, em áreas gerais, como Clínica Médica Geral, Cirurgia Geral ou Saúde Pública ${ }^{(22)}$.

Por outro lado, a sociedade brasileira, que procura, em grande parte, espelhar-se na estadunidense, 
responde com considerável retarde às transformações que lá ocorrem. Nos últimos anos, tem sido notável o investimento da sociedade norte-americana em mudanças na organização do trabalho médico, de modo a resultar em que uma proporção crescente dos mesmos esteja engajada profissionalmente na atenção primária à saúde e no trabalho junto às famílias e à comunidade ${ }^{(20,21,23)}$. No entanto, é previsível que estas transformações atinjam a sociedade brasileira com uma demora de vários anos, ou, talvez, de décadas. Neste momento, será possível vislumbrar a existência de um forte programa de treinamento pós-graduado, na área de Residência Médica, em Medicina Geral Comunitária, com características multidisciplinares, no qual se destaquem centros de excelência ligados às principais universidades brasileiras. Os egressos destes programas disputarão posições de prestígio e de alta remuneração em unidades básicas do sistema nacional de saúde, ou, mesmo, em empresas privadas de assistência médica, convencidas das vantagens do investimento em atenção primária e na promoção da saúde. Neste cenário, não é difícil imaginar que a mudança do modelo de ensino clínico hospitalar para o comunitário ocorrerá de modo natural, livre das dificuldades que hoje se apresentam.

\section{DIFICULDADES A IMPLEMENTAÇÃO}

A mudança de parte substancial do ensino médico de graduação, no campo clínico, do hospital universitário para os serviços de saúde na comunidade apresenta, no momento atual brasileiro, um número de dificuldades de ordem prática. A alta amplitude destas dificuldades constitui, também, uma das barreiras à aceitação desta idéia, que se ajunta às acima comentadas.

A maior parte destas dificuldades consiste, exatamente, da falta, na maior parte dos municípios onde se localizam as escolas médicas brasileiras, dos requisitos básicos ao bom desenvolvimento do ensino clínico na rede de serviços de saúde da comunidade, anteriormente descritos (Tabela III). O número de unidades funcionantes e a abrangência geográfica da rede são, ainda, acentuadamente reduzidos e, aparentemente, estão longe de cobrir as necessidades do público. A organização do sistema, compreendendo, neste ponto, a devida interação entre as esferas municipal, estadual e federal é, também deficiente, apesar dos grandes avanços da recente reorganização do sistema.
Um fator limitante da maior importância é a relativa ineficiência do conjunto das unidades básicas de saúde, notadamente no que se refere à resolutividade dos casos que demandam assistência médica; disto resulta acentuado prejuízo à imagem do serviço junto ao público e junto à própria comunidade médica, que inclui professores e estudantes de Medicina. É provável que isto decorra da falta de um maior número de médicos com treinamento específico e capacitação técnica apropriada para exercer a Medicina Geral Comunitária. É fato bem conhecido que o exercício de atividades gerais por especialistas resulta em baixa resolutividade e alto percentual de encaminhamento, a outros profissionais, daqueles casos que não se enquadram na especialidade do médico que faz o atendimento primário ${ }^{(23)}$. Ajunta-se a isto a baixa motivação de médicos precariamente remunerados e com grau muito reduzido de compromisso, pessoal com as instituições em que trabalham. Neste contexto, é altamente previsível que tais profissionais terão, também, pouca motivação para trabalharem com estudantes, além de lhes faltar, ainda, um mínimo de capacitação na esfera educacional ${ }^{(24)}$. É duvidoso, também, que, nestas circunstâncias, possa ser encontrada, entre os profissionais que militam na rede de serviços à comunidade, alta prevalência de "role models" apropriados em quem os estudantes de Medicina possam se espelhar ${ }^{(15,25)}$.

Faltam, por fim, os necessários vínculos institucionais, estáveis e efetivos, entre a escola médica, de um lado, e a rede de serviços de saúde na comunidade, de outro, para que as questões administrativas e de planejamento e desenvolvimento educacional possam ser devidamente equacionadas.

\section{CONSIDERAÇÕES FINAIS}

O desenvolvimento de parte considerável do ensino médico de graduação, na área clínica, fora do hospital universitário e junto aos serviços de saúde da comunidade, visando à formação de médicos com características mais adequadas ao atendimento das necessidades da sociedade, sofre grande resistência no seio das escolas médicas e enfrenta dificuldades práticas de implementação. A superação dos obstáculos dar-se-á, possivelmente, pela conjunção de várias forças e exigirá grande empenho daqueles que estão convencidos da pertinência desta transformação.

Embora a difusão deste conceito, no seio da escola médica, e a necessária adequação da rede de 
serviços de saúde, na comunidade, constituam tarefas das mais difíceis, as forças que atuam no sentido de mudança podem ser ajudadas pela inviabilização progressiva do hospital de ensino e por exigências crescentes da sociedade para que haja substituição do paradigma tradicional de atuação médica. Cabe, po- rém, ao Estado e às entidades ligadas à profissão médica um papel extremamente importante. Medidas efetivas de apoio ao ensino clínico na comunidade devem contemplar o fortalecimento do sistema nacional de saúde e a devida valorização profissional do trabalho dos médicos junto à comunidade.

TRONCON LEA. Clinical teaching in community health services. Medicina, Ribeirão Preto, 32: 335-344, july/sept. 1999.

ABSTRACT: Moving undergraduate clinical teaching from university hospitals to communitybased services is currently accepted as a strong trend in Medical Education. The main purpose of this change is to build a new model of medical action, which is though to be more suitable to public needs. Community-oriented clinical teaching is justifiable not only as the basis of this new model, but also on educational grounds, since it is thought to have many potencial advantages regarding student learning. There are, however, several cultural factors opposing the acceptance of community-oriented clinical teaching within medical schools. Also, the implementation of the relevant student activities is made difficult by a number of practical reasons. Breaking barriers and overcoming difficulties is a complex task, which should involve medical schools as well as the government and medical councils and associations. Effective actions in support of communitybased clinical teaching must include the strenghtening of the national health system and the valorization of physician's work in community services.

UNITERMS: Students, Medical. Schools, Medical. Community Network. Community Health Services. Education, Medical.

\section{REFERÊNCIAS BIBLIOGRÁFICAS}

1 - HARDEN RM; SNOWDEN S \& DUNN WR. Educational strategies in curriculum development: the SPICES model. Med Educ 18: 284-297, 1984

2 - LOWRY S. Trends in health care and their effects on medical education. BMJ 306: 255-258, 1992.

3 - OLIVEIRA HL. Medical education and development. World Health Organization Regional Office for the Eastern Mediterraneum, EMRO/71/370, 1971.

4 - ABATH GM. Medicina geral comunitária no Brasil. Rev Bras Educ Méd 8: 166-176, 1984.

5 - CHAVES MM. Educação das profissões da Saúde: perspectivas para o século XXI. Rev Bras Educ Méd 20: 21-28, 1996.

6 - BARROWS HS. Practice-based learning. Problem-based learning applied to medical education. Southern Illinois University School of Medicine, Springfield, Illinois, 1994.

7 - NEUFELD VR; WOODWARD CA \& MACLEOD SM. The McMaster MD program; a case study in renewal in Medical Education. Acad Med 64: 423-432, 1989.
8 - MOORE GT; BLOCK SD; STYLE CB \& MITCHELL R. The influence of the new pathway curriculum on Harvard medical students. Acad Med 69: 983-989, 1994.

9 - ANDERSON J \& GRAHAM A. A problem in medical education: is there an information overload?. Med Educ 14: 4-7, 1980.

10 - FERREIRA JR. Formação de recursos humanos para a medicina do ano 2000. Rev Bras Educ Méd 7: 93-100, 1983.

11 - NEWBLE DI \& ENTWISTLE NJ. Learning styles and approaches: implications for medical education. Med Educ 20: 162-175, 1:986.

12 - REES L \& WASS J. Undergraduate medical education. BMJ 306: 258-261, 1993.

13 - LOWRY S. Curriculum design. BMJ 305: 1409-1411, 1992.

14 - KAUFMAN A; MENNIM S; WATERMAN R; DUBAN S; HANSBERGER C; SILVERBLATT H; OBENSHAIN SS; KANTEROWITZ M; BECKER T \& SANNET J. The New Mexico experiment: educational innovation and institutional change. Acad Med: 64 285-294, 1989. 
15 - FICKLIN FL; BROWNE VL; POWEL RC \& CARTER JE. Faculty and house staff members as role models. $\mathbf{J}$ Med Educ 63 : 392-396, 1988.

16 - QUEIROZ MS. Perspectivas teóricas sobre medicina e profissão médica: uma proposta de enfoque antropológico. Rev Saude Publ 25: 318-325, 1991.

17 - STEINER E \& STOKEN JM. Overcoming barriers to generalism in Medicine; the residents' perspective. Acad Med 70: S89-S96, 1995, Suppl.January

18 - AMORIM DS. Óbice à reorganização curricular: o departamento (carta). Rev Bras Educ Méd 17: 40, 1993.

19 - AMORIM DS. The archaic organization of the higher education institutes and its effects on the curriculum. Rev Bras Educ Méd 19: 12-15, 1995.

20 - ASSOCIATION OFAMERICAN MEDICAL COLLEGES. AAMC policy on the Generalist Physician. Acad Med 68: 1-6, 1993.

21 - O'NEIL EH \& SEIFER S. Health care reform and medical education: forces toward Generalism. Acad Med 70: S37S43, 1995, Suppl. January.
22 - TRONCON LEA; CIANFLONE ARL; RODRIGUES MLV; PICINATO CE, PERES LC \& FIGUEIREDO JFC. Avaliação terminal de competências clínicas dos graduandos em Medicina: relato da experiência inicial da Faculdade de Medicina de Ribeirão Preto, Faculdade de Medicina de Ribeirão Preto da USP, Ribeirão Preto, 1996.

23 - GABRIEL SE. Primary care: specialists or generalists. Mayo Clin Proc 71: 415-419, 1996.

24 - HITCHCOCK MA, STRITTER FT \& BLAND CJ. Faculty development in the health professions: conclusions and recomendations. Med Teach 14; 295-309, 1993.

25. SKEFF KM \& MUTHA S. Role models: finding the future of medicine. N England J Med 339: 2015-2017, 1998.

Recebido para publicação em 17/03/99

Aprovado para publicação em 14/09/99 